\title{
LE RAPPORT TROMPE/PALPES DANS LES BIOTYPES \\ DU COMPLEXE CULEX PIPIENS ET LEURS HYBRIDES
}

\author{
Par J. CALLOT
}

Parmi les caractères qui ont été donnés pour distinguer morphologiquement les adultes du Culex pipiens de ceux du Culex autogenicus, un des moins mauvais est tiré du rapport entre la longueur de la trompe du mâle et de celle des quatre premiers articles des palpes maxillaires.

Les auteurs qui ont étudié ee caractère concluent en disant que chez pipiens la trompe est généralement plus courte que l'ensemble des quatre premiers articles des palpes maxillaires, alors que chez autogenicus elle est généralement un peu plus longue.

Il nous a paru intéressant de rechercher comment se comportait exactement ce caractère, soit chez l'un ou l'autre de ces biotypes, soit dans le produit de leur croisement.

$$
*
$$

La trompe est très généralement moins longue que les quatre premiers articles des palpes maxillaires chez des Culex pipiens présentant les propriétés biologiques d'eurygamie, d'hétérodynamie, d'anautogénèse et d'ornithophilie, la coloration sombre du thorax et dont l'abdomen est, sur sa face sternale, largement marqué d'écailles sombres. C'est du moins ce que je relève dans mes notes sur les Culex des environs de Strasbourg, de Richelieu, du SudOuest de la France.

Dans une série examinée récemment et provenant des environs de Strasbourg je trouve pour 20 mâles une moyenne de 72,1 $\sigma 3,12$ divisions micrométriques (1) pour la trompe et de 76,5 $\sigma \mathbf{2 , 4 2}$ divisions pour l'ensemble des quatre premiers articles des palpes, soit un rapport de 100 à 106 . Le calcul des pourcentages donne approximativement :

(1) De $0,03 \mathrm{~mm}$.

Ann. de PARasitologie, T. XXIX, N ${ }^{\circ} 1-2 .-1954$. 
palpes plus longs $\ldots \ldots \ldots \ldots \ldots \ldots \ldots \ldots .85 \%$

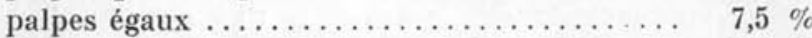

palpes plus courts $\ldots \ldots \ldots \ldots \ldots \ldots \ldots \ldots \ldots \ldots \ldots \ldots$

Une souche autogène provenant d'une fosse septique de Strasbourg nous donne les résultats suivants pour un même nombre de mâles : trompe M. $74,3 \sigma 2,69$ divisions ; palpes, $70 \sigma 2,10$. Le rapport est donc inverse de 100 à 95 , avec la répartition suivante :

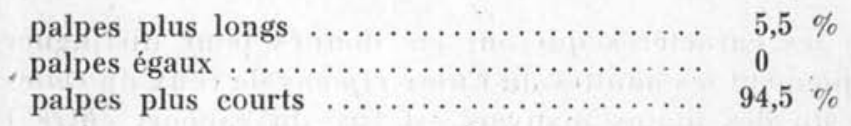

Une autre souche ayant les mêmes caractères biologiques, obtenue à partir d'une femelle capturée alors qu'elle se gorgeait sur l'homme dans le Sud-Ouest (Charente-Maritime), montre à la seconde génération les caractères suivants sur deux pontes : trompe M. 69 et $74,8 \sigma 3,45$ divisions ; palpes M. 67,5 et $71,6 \sigma 2,23$, soit les rapports de 100 à 97 et 100 à 96 avec les pourcentages suivants pour les deux lots réunis :

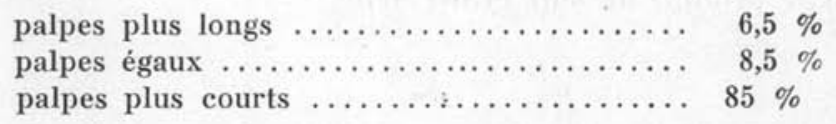

Ces résultats sont donc comparables aux données précédentes.

On peut en conclure que chez le Culex anautogène, eurygame et hétérodyname, l'extrémité du quatrième article des palpes maxillaires dépasse, mais légèrement, l'extrémité de la trompe dans $85 \%$ des cas au moins, alors que chez le biotype autogène, sténogame, homodyname dans plus de $85 \%$ des cas, on constate l'inverse.

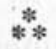

Le croisement des femelles autogènes avec des mâles du type pipiens ci-dessus défini nous a donné les résultats suivants, à l'examen morphologique de la première génération d'hybrides.

Les mâles présentent l'apparence de mâles de pipiens, avec une coloration générale sombre et une ornementation sternale très nette. Sur 32 mâles examinés, on obtient, quant aux rapports entre palpes 
et trompe, un lot avec une moyenne de $74,4 \sigma 1,68$ pour la trompe et $74 \sigma 2,52$ divisions pour les palpes, l'autre avec 72,58 $\sigma 2,30$ pour la trompe et $72,24 \sigma 2,52$ pour les palpes, soit un rapport de 100 à 99,5 avec la répartition suivante :

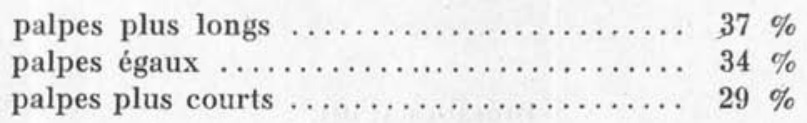

Il y a donc une répartition à peu près équivalente entre les trois possibilités.

Ces hybrides qui, comme c'est la règle, ne présentaient pas le caractère d'autogénèse, mais qui ont été nourris sur un poulet, donnèrent une progéniture dans laquelle les mâles avaient le type paternel et les femelles avaient aussi, mais pas toutes, le type pipiens.

La mensuration des trompes et des palpes donne les indications suivantes : pour 17 mâles d'une ponte, trompe : 82,65 $\sigma 2,21$; palpes : $81,8 \sigma 2,78$, soit 100 à 99 . Une autre ponte donne pour 19 mâles, trompe : $71,5 \sigma 1,94$; palpes : $72,2 \sigma 1,94$ avec le rapport 100 à 101 et une troisième ponte avec aussi 19 mâles, trompe : 78,78 $\sigma 1,88$, palpes : $78,57 \sigma 2,04$ avec le rapport 100 à 99,75 environ et la répartition générale suivante pour les 55 mâles :

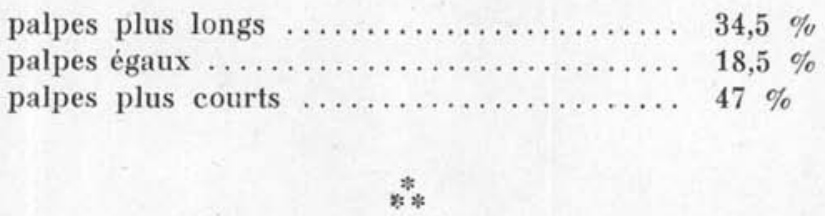

Les mensurations des palpes et des trompes offrent dans leur apparente simplicité quelques difficultés. Peu pratiques sur les insectes piqués, elles risquent, sur des exemplaires montés à la gomme au chloral, d'être rendues approximatives par l'obliquité des palpes dans le plan vertical et par la fréquente coudure des trompes:

Cependant, compte tenu de ces réserves et en ne faisant pas dire aux chiffres plus qu'ils ne peuvent dire, on peut en conclure que :

$1^{\circ}$ Chez Culex pipiens L. sensu stricto, le quatrième article du palpe du mâle dépasse l'extrémité des labelles dans au moins $85 \%$ des cas.

$2^{\circ}$ Dans le cas du biotype Culex autogenicus $\mathrm{R}$. (= molestus auct.) 
la trompe dépasse l'extrémité distale du quatrième article des palpes maxillaires des mâles dans $90 \%$ des exemplaires au moins.

$3^{\circ}$ Dans le cas des hybrides de première génération, on trouve que la trompe atteint, n’atteint pas ou dépasse cette extrémité, dans un nombre égal de cas.

\section{BIBLIOGRAPHIE}

Mattingly (P. F.) et coll. - The Culex pipiens complex. Trans. Roy. Ent. Soc. London, CII, 1951, p. 331-382.

(Institut de Parasitologie, Faculté de Médecine, Strashourg). 